Supporting Information

\title{
Scattering Form Factor of Block Copolymer Micelles with Corona Chains Discretely Distributed on the Core Surface
}

\author{
Rintaro Takahashi ${ }^{* \dagger}$ Shota Fujii, ${ }^{\dagger}$ Isamu Akiba, ${ }^{\dagger}$ Kazuo Sakurai ${ }^{* \dagger}$ \\ 'Department Chemistry and Biochemistry, University of Kitakyushu, 1-1 Hibikino, \\ Wakamatsu-ku, Kitakyushu, Fukuoka, 808-0135, Japan \\ *(R.T.)E-mail:r-takahashi@kitakyu-u.ac.jp, (K.S.)E-mail:sakurai@kitakyu-u.ac.jp.
}

\section{Table of Contents}

Derivation of the PG model in the consideration of scattering from many micelles...pp S2 Is $F_{\text {corona }}(q)$ influenced by whether the Tammes problem or not? (Figure S1).......pp S3 Each term of $F_{\text {corona }}(q)$ in PG model (Figure S2)............................................pp S3 $F_{\text {corona }}(q)$ with the separately-varied $R$ and $R_{\mathrm{g}}$ values (Figure S3) ........................pp S4 


\section{Derivation of the PG model in the consideration of scattering from many micelles.}

To proceed with the calculation using eq 11, we need to know the radial distribution function of the center of mass of the $j$-th corona chain $\left[P_{j}(\theta, \phi)\right]$, assuming that each corona chain is identical. This means that $P_{j}(\theta, \phi)$ does not depend on how the $i$-th chain is chosen.

$$
\sum_{i}^{N} \sum_{j(i \neq j)}^{N} \frac{\sin \left(q r_{i j}\right)}{q r_{i j}}=\frac{1}{2} N \sum_{j=2}^{N} \int_{0}^{\pi} \int_{0}^{2 \pi} P_{j}(\theta, \phi) \frac{\sin \left(q r_{1 j}\right)}{q r_{1 j}} d \Omega
$$

Here $d \Omega$ is the differential solid angle given by $d \Omega=\sin \theta d \theta d \phi$ and $\int_{0}^{\pi} \int_{0}^{2 \pi} P_{j}(\theta, \phi) d \Omega=1 . P_{j}(\theta, \phi)$ indicating the probability of finding the center of mass of the $j$-th chain at the solid angle of $\Omega$. If we can assume that the chains are randomly distributed on the core surface (this seems to contradict the even distribution), we have $P_{j}(\theta, \phi)=1 /(4 \pi)$. Note that $r_{1 j}$ is expressed as $r_{1 j}=2\left(R+R_{\mathrm{g}}\right) \sin (\theta / 2)$ depending only on $\theta$. Therefore, eq S1 becomes

$$
\begin{aligned}
\sum_{i}^{N} \sum_{j(i \neq j)}^{N} \frac{\sin \left(q r_{i j}\right)}{q r_{i j}}=\frac{1}{4 \pi} & \frac{1}{2} N(N-1) 2 \pi \int_{0}^{\pi} d \theta_{i j} \frac{2 \sin \left\{q\left[2\left(R+R_{\mathrm{g}}\right) \sin \frac{\theta_{i j}}{2}\right]\right\}}{q\left[2\left(R+R_{\mathrm{g}}\right) \sin \frac{\theta_{i j}}{2}\right]} \sin \theta_{i j} \\
& =N(N-1)\left\{\frac{\sin \left[q\left(R+R_{\mathrm{g}}\right)\right]}{q\left(R+R_{\mathrm{g}}\right)}\right\}^{2}
\end{aligned}
$$

After adjusting $N$ and $N_{\text {agg }}$ in eqs 4 and 13, we can obtain eq 6 . That is, in the case of many micelles, the PG model assumes that $P_{j}(\theta, \phi)=1 /(4 \pi)$. This assumption means that the corona chains are randomly distributed on the core surface. Hence, in deriving the PG model, the different assumptions are needed for a single micelle scattering and many micelles scattering. Pedersen ${ }^{\mathrm{S}}$ referred to the assumption as evenly distributed corona chains. In either case, the interference effect between the corona chains is not taken into account. 


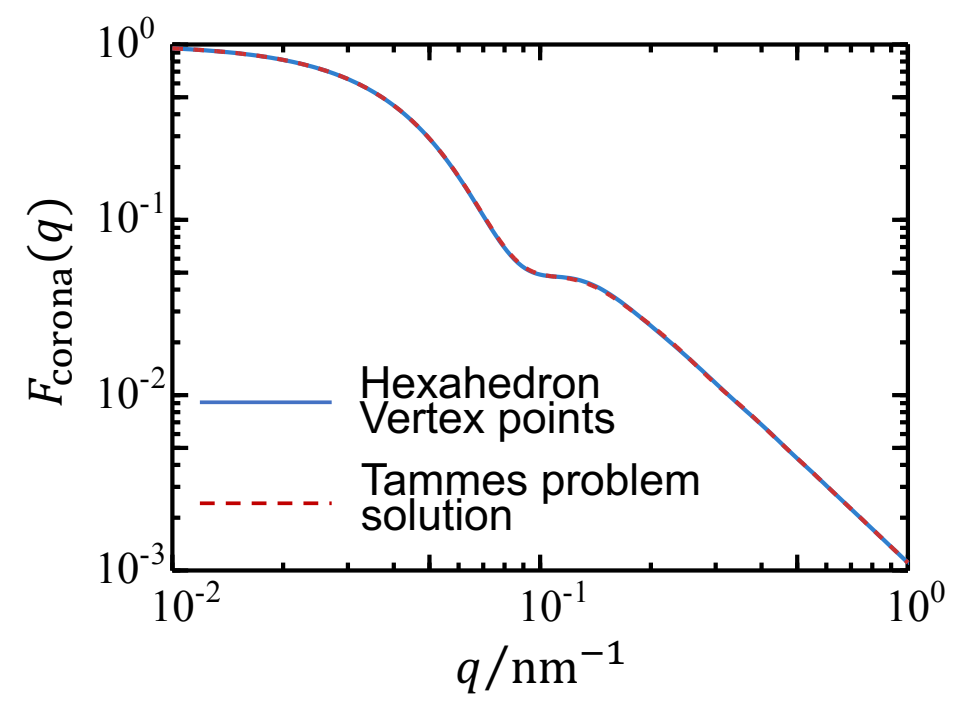

Figure S1. $F_{\text {corona }}(q)$ calculated using the vertex points of the inscribed hexahedron (solid solid curve) and the solution of the Tammes problem (broken red curve) in $N_{\text {agg }}$ $=8$ and $R=R_{\mathrm{g}}=15 \mathrm{~nm}\left(\sigma_{\text {corona }}=0.5\right)$.

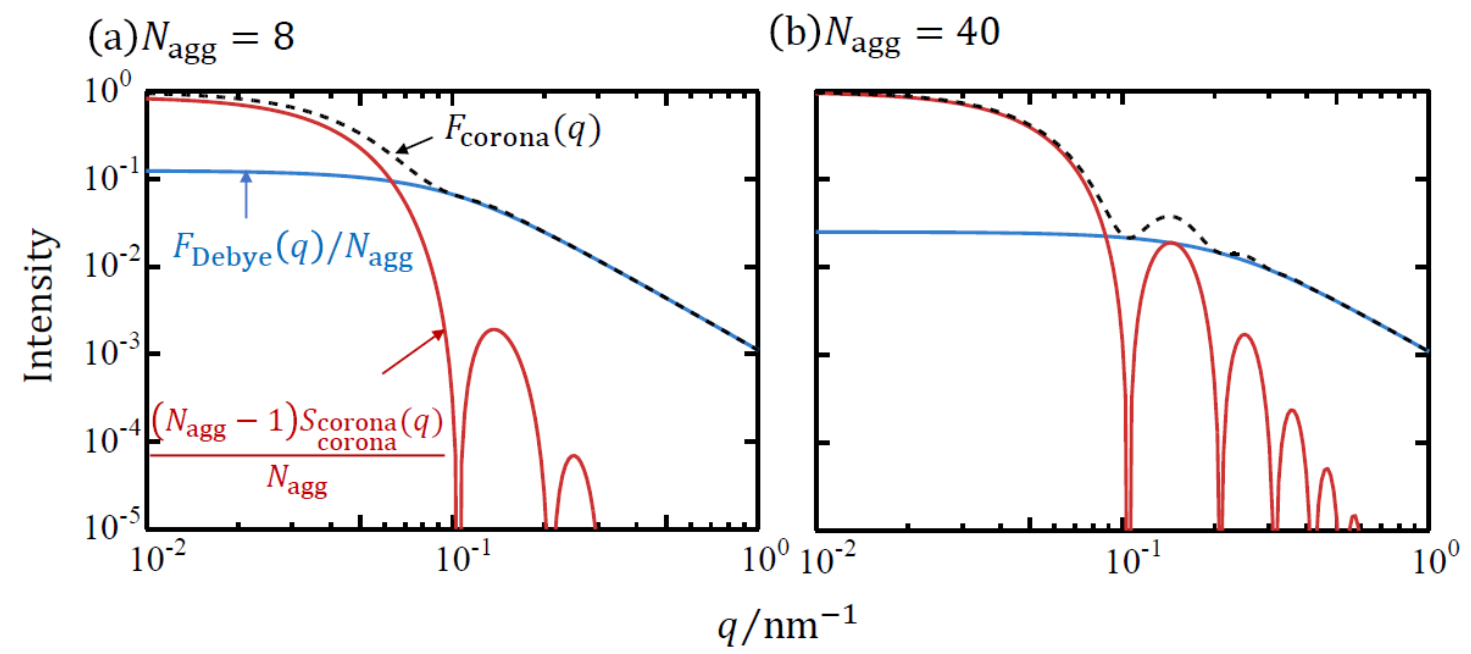

Figure S2. Each term of $F_{\text {corona }}(q)$ in the PG model in $N_{\text {agg }}=8$ (a) and 40 (b) under the $R+R_{\mathrm{g}}$ value of $30 \mathrm{~nm}$ and $\sigma_{\text {corona }}$ value of 0.5 . The solid blue curve represents $F_{\text {Debye }}(q) / N_{\text {agg }}$, the solid red curve is $\left(N_{\text {agg }}-1\right) S_{\text {corona }}(q) / N_{\text {agg }}$, and the broken black curve is $F_{\text {corona }}(q)$. 


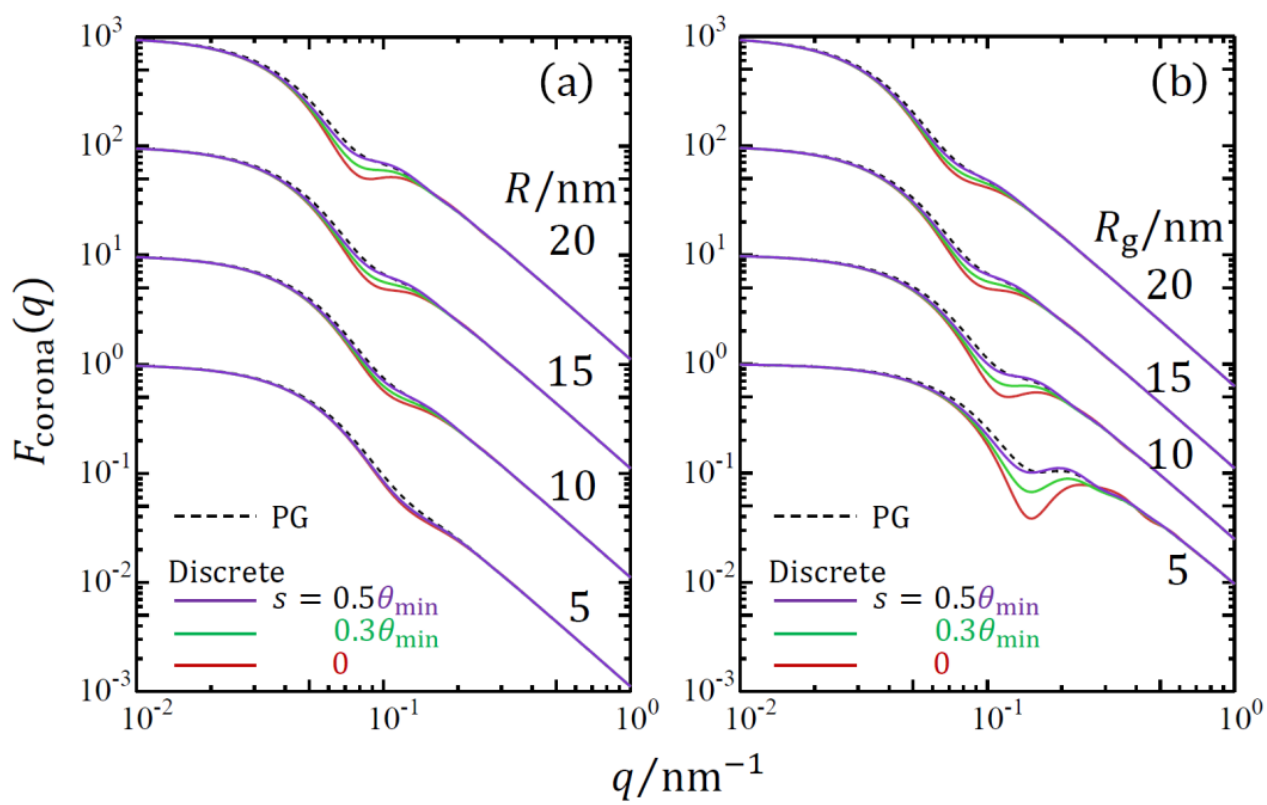

Figure S3. $F_{\text {corona }}(q)$ in $N_{\text {agg }}=8$ for the various $R$ values of $5,10,15$, and $20 \mathrm{~nm}$ under the constant $R_{\mathrm{g}}$ value of $15 \mathrm{~nm}$ (a) and for the various $R_{\mathrm{g}}$ values of 5, 10, 15, and $20 \mathrm{~nm}$ under the constant $R$ value of $15 \mathrm{~nm}$ (b). The broken black curve represents the PG model. The solid red, green, and purple curves indicate our proposed discrete model with and without the distortion $\left(s=0,0.3 \theta_{\min }\right.$, and $0.5 \theta_{\min }$, respectively). The curves are shifted vertically for clarity.

\section{Reference}

(S1) Pedersen, J. S. Structure Factors Effects in Small-angle Scattering from Block Copolymer Micelles and Star Polymers. J. Chem. Phys. 2001, 114, 2839-2846. 